Exercices Exercices de rhétorique

de rhétorique $\quad 7 \mid 2016$

Les rhétoriques du peuple

\title{
Les Deux Méthodes : entre interdiscours et interlocution
}

Émilie Goin et François Provenzano

\section{OpenEdition}

1 Journals

Édition électronique

URL : http://journals.openedition.org/rhetorique/479

DOI : $10.4000 /$ rhetorique.479

ISSN : 2270-6909

Éditeur

UGA Éditions/Université Grenoble Alpes

Édition imprimée

ISBN : 978-2-37747-084-6

Référence électronique

Émilie Goin et François Provenzano, «Les Deux Méthodes : entre interdiscours et interlocution »,

Exercices de rhétorique [En ligne], 7 | 2016, mis en ligne le 19 juin 2016, consulté le 12 septembre 2020.

URL : http://journals.openedition.org/rhetorique/479; DOI : https://doi.org/10.4000/rhetorique.479

Ce document a été généré automatiquement le 12 septembre 2020.

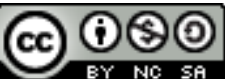

Les contenus de la revue Exercices de rhétorique sont mis à disposition selon les termes de la Licence Creative Commons Attribution - Pas d'Utilisation Commerciale - Partage dans les Mêmes Conditions

4.0 International. 


\title{
Les Deux Méthodes : entre interdiscours et interlocution
}

\author{
Émilie Goin et François Provenzano
}

1 La brochure publiée en 1900 par l'Imprimerie Ouvrière P. Lagrange de Lille sous le titre Les Deux Méthodes offre une transcription - augmentée d'intertitres, des interventions de l'auditoire et du modérateur - des discours prononcés successivement par les deux leaders socialistes Jean Jaurès et Jules Guesde lors d'une « conférence » contradictoire organisée en novembre de la même année à l'Hippodrome lillois. L'analyse contrastive que nous voulons mener de ces deux discours visera, pour l'essentiel, à mettre en lumière "deux méthodes " rhétoriques distinctes dans la gestion des phénomènes interdiscursifs et interlocutifs. Ces « deux méthodes » seront par ailleurs mises en lien avec des imaginaires du peuple et, plus particulièrement, avec la place donnée à ces imaginaires dans un dispositif de parole publique.

2 Avant d'entrer dans le détail de cette analyse, nous contextualiserons rapidement la portée politique dudit dispositif - celui de la conférence contradictoire-, nous rappellerons ensuite quelques éléments biographiques sur les contradicteurs, propres à éclairer leur ethos préalable, nous préciserons enfin en quoi notre hypothèse de travail permet de prolonger et d'approfondir les analyses menées sur le même corpus, dans la tradition de l'analyse du discours, par Pierre Muller ${ }^{1}$, qui s'est quant à lui centré prioritairement sur la dynamique interlocutive, en associant les postures énonciatives des deux contradicteurs à leur positionnement idéologique. Nous tenterons, pour notre part, de montrer en quoi la prise en compte des faits d'interdiscours permet d'affiner ces résultats. Nous viserons aussi à dégager la place faite au peuple-public de la réunion au sein de ce débat, de même que la manière dont le public investit cette place, et enfin la manière dont les réactions du public sont gérées par chaque orateur. Nous serons attentifs aux phénomènes d'adresse, en particulier l'usage des pronoms inclusifs (on, nous, vous, eux), ainsi qu'aux imaginaires mobilisés dans la construction de l'ethos des orateurs et des images de l'orateur adversaire et du peuple-public. 


\section{Le genre de la conférence contradictoire et sa portée politique}

\subsection{La réunion politique comme renforcement du régime représentatif}

3 À une époque encore hantée par le spectre de la révolution, où les rassemblements sur la voie publique sont interdits, la loi du 30 juin 1881 autorise l'organisation de réunions dans des lieux cadrés. Paula Cossart interroge le statut de ces réunions au tournant du $\mathrm{XIX}^{\mathrm{e}}$ et du XXe $\mathrm{Xiècle}^{2}$. Encouragée par les républicains, la réunion politique est présentée par eux comme un mode démocratique d'expression, une forme de participation, qui permet de compléter le vote sans risquer de concurrencer et de fragiliser le régime représentatif et sans menacer l'ordre public. À l'action subversive qui résultait des rassemblements sur la voie publique, se substitue le débat dans une salle de réunion, menant à la constitution d'une opinion publique.

Pour les républicains du dernier tiers du $\mathrm{XIX}^{\mathrm{e}}$ siècle, encore influencés par les conceptions du XVIII (Montesquieu, Rousseau, Necker), cette opinion doit émerger d'une discussion rationnelle pour aboutir à une forme de consensus ayant pour objectif le bien commun. Cette opinion calculée en fonction de "l'intérêt général » serait par définition stable et unifiée ${ }^{3}$. Selon Roger Chartier, « elle est donc l'inverse de l'opinion populaire, multiple, versatile, habitée par les préjugés et les passions ${ }^{4}$ ». À la différence du XVIII ${ }^{e}$ siècle toutefois, l'assemblée ne serait plus seulement constituée d'une élite de citoyens éduqués, mais de l'ensemble des citoyens électeurs ${ }^{5}$.

5 Pour pouvoir devenir des citoyens acteurs de la démocratie, les individus doivent cependant, aux yeux des républicains du XIX ${ }^{\mathrm{e}}$, être formés. Cette formation à la citoyenneté repose principalement sur une maitrise de la manifestation des émotions dans l'espace public, ne rendant acceptables que les comportements raisonnés et tempérés. Ce faisant, elle convoque l'imaginaire romantique bien connu du peuple dominé par ses passions. Pourtant, l'émotion n'est pas totalement exclue de l'espace public. Dans la conception républicaine en particulier, la réunion est considérée comme une forme d'exutoire : il n'y a rien à craindre des discours violents et/ou annonçant une révolution prochaine, tant qu'ils restent circonscrits dans le cadre de la salle de réunion et qu'ils ne sont pas une incitation directe à l'action violente ${ }^{6}$. Un « régime émotionnel spécifique ${ }^{7}$ » est alors institué : des «normes émotionnelles » sont posées, certaines émotions (celles des orateurs) étant acceptées tandis que d'autres (celles du public) sont sanctionnées ${ }^{8}$. La violence devient ainsi un monopole de l'État, tandis que le citoyen est généralement appelé à manifester « une adhésion silencieuse par laquelle s'établit rituellement mais pacifiquement le mécanisme politique de la délégation ${ }^{9}$ ». La réunion est ainsi perçue par les républicains comme un moyen d'obtenir une «pacification des modes collectifs d'expression politique» et, à travers elle, une pacification des mœurs ${ }^{10}$. L'impératif d'ordre et de modération lors des réunions est d'autant plus essentiel pour les républicains que, depuis le vote de la loi de 1881, les assemblées formées lors des réunions sont considérées comme l'incarnation de la République. Dès lors, dès qu'une réunion tourne mal (bagarres, interruptions empêchant les orateurs de parler), la presse impérialiste présente ces incidents comme le signe de l'échec de la République et de la menace révolutionnaire ${ }^{11}$. Cossart ajoute 
que, du côté des organisateurs des réunions, il est fréquent que les fauteurs de trouble soient dénoncés comme étant des agents du Gouvernement.

6 Selon elle, les pratiques promues par les républicains ne sont pas simplement absorbées ou rejetées par le peuple (en l'occurrence, par le public des réunions). Au contraire «le peuple va jouer avec les normes, se les approprier, les subvertir, [...] cette réception peut faire évoluer la définition même de ce qui constitue un bon comportement politique dans une démocratie représentative ${ }^{12}$ ». Ainsi Cossart explique que si le régime émotionnel imposé par les organisateurs des réunions crée bien un contraste entre la violence des propos tenus par les orateurs et le calme de l'assemblée réunie dans la salle, c'est également parce que le public, en grande partie composé de curieux, n'appréhende pas toujours les discours des orateurs (en particulier les thèses révolutionnaires et anarchistes) avec sérieux, mais plutôt comme une forme de spectacle ; les rires sont ainsi très fréquents dans la salle ${ }^{13}$.

7 Sous le Second Empire, le Gouvernement impérial estime qu'aucune opinion raisonnée (sous-entendue modérée ou conservatrice) ne peut émaner des réunions politiques largement dominées par des passions (sous-entendues révolutionnaires). Selon eux, le peuple rassemblé est enclin à suivre l'orateur qui utilisera au mieux le pathos. Les républicains estiment au contraire que dans une discussion libre, c'est généralement les meilleurs arguments qui triomphent. Dans cette optique, il est nécessaire pour eux que les réunions rassemblent en un même lieu plusieurs personnes défendant des idées diverses de manière à ce que, par le libre jeu de la contradiction, soient disqualifiées les idées extrêmes ainsi que les idées ne représentant l'intérêt que d'un groupe, au profit d'idées visant le bien commun ${ }^{14}$. Ainsi, «le discours républicain sur les vertus de la délibération des citoyens en réunion est marqué par un appel à la recherche du bien commun, impliquant alors un rejet de l'idéologie qui ne refléterait que des intérêts particuliers ${ }^{15} »$. Cette conception républicaine d'une délibération ayant pour visée l'intérêt général prend la forme d'un rejet des opinions jugées extrêmes, qu'elles soient révolutionnaires, socialistes ou anarchistes. Sous l'Empire déjà, la presse républicaine dénonçait les réunions socialistes comme empêchant la libre contradiction de se dérouler jusqu'à son terme, laquelle finirait par démontrer l'erreur socialiste. L'idée de l'intérêt général mène à disqualifier toute protestation ou contestation, en particulier si celles-ci sont exprimées avec passion. Selon Cossart, si les républicains défendent autant les vertus de la délibération, c'est parce qu'ils veulent avant tout consolider la démocratie représentative, en supprimant tout ce qui pourrait la menacer ${ }^{16}$. Le rôle des citoyens dans le processus de délibération est également tempéré: "il ne s'agit nullement alors d'associer les citoyens à la prise de décision en remettant en partie celle-ci à leur assemblées. Il s'agit simplement de voir s'y former une opinion publique orientée vers le bien commun ${ }^{17}$ ». Il faut ajouter que l'opinion ainsi développée est indépendante du gouvernement représentatif; elle ne remonte jamais jusqu'à lui. Les socialistes défendront plus tard l'idée qu'il est nécessaire d'associer la délibération des citoyens en réunion à l'initiative parlementaire, mais elle ne sera jamais réalisée, pas même de nos jours où l'influence des dispositifs participatifs et délibératifs sur les décisions des organismes gouvernementaux n'est pas démontrée ${ }^{18}$.

8 En somme, la réunion politique a pour objectif la pacification des mœurs et la formation d'une opinion publique unifiée; elle permet d'exclure l'ensemble des pratiques jugées subversives (action sur la voie publique, appels à l'action, idées révolutionnaires) et contribue ainsi au renforcement du régime représentatif. 


\subsection{La conférence contradictoire : une polémique bien encadrée}

9 L'influence républicaine explique vraisemblablement pourquoi la contradiction est devenue la scénographie la plus fréquente des réunions politiques dans la culture rhétorique française des premières décennies de la III $^{e}$ République. Mais elle fait toutefois l'objet de critiques qui l'assimilent à une cause de désordres et de violences. Pour contrer les éventuels débordements, l'organisation de ces assemblées prévoit un bureau et un président chargés de maintenir le calme de manière à ce que les deux discours contraires puissent se faire entendre ${ }^{19}$. La loi de 1881 concernant le rôle du bureau décrit ce dernier comme étant chargé « d'interdire tout discours contraire à l'ordre public et aux bonnes mœurs, ou contenant provocation à un acte qualifié crime ou délit ${ }^{20}$ ». Il est également chargé de « faire respecter le caractère qui a été donné à la réunion, soit par la déclaration, soit par la convocation ou les affiches ${ }^{21} »$; ce qui signifie, pour une conférence contradictoire, que le bureau doit assurer la possibilité aux contradicteurs de répondre librement aux orateurs ${ }^{22}$. La tâche est toutefois difficile pour le bureau qui, pour ce faire, n'a à sa disposition que des « moyens moraux ${ }^{23} »$. Les membres du bureau

[...] doivent avant tout user de l'autorité morale que leur donne le mandat qu'ils ont reçu, maintenir la régulation et la dignité des discussions par des exhortations et des remontrances adressées aux interrupteurs, ramener le calme au moyen de rappels à l'ordre infligés aux perturbateurs ${ }^{24}$.

Pourtant les interventions libres du public sont ce qui fait de la réunion contradictoire la forme la plus légitime de réunion à cette période. Ceux qui n'en organisent pas - leur préférant des réunions privées où la contradiction n'est pas admise - sont considérés comme des lâches qui craignent la critique et préfèrent «faire la leçon au peuple ${ }^{25}$ ». Cossart souligne que ce type de dénonciation est particulièrement fréquent, trente ans plus tard, lors de l'affaire Dreyfus: les dreyfusards critiquent leurs adversaires qui, contrairement à eux, tiennent seulement des réunions fermées ${ }^{26}$. Dans cet ordre d'idées, il est bien vu pour un orateur de montrer qu'il ne craint pas d'être contredit, voire qu'il désire la contradiction. Il est ainsi fréquent qu'un orateur insiste sur sa disposition à recevoir la critique (en particulier quand il sait que le public lui est acquis $^{27}$ ).

11 Il est intéressant de noter que les discours a priori agonistiques tenus lors de ces réunions contradictoires présentent fréquemment une série de caractéristiques communes. Dans le cas qui nous occupe ici, Jaurès et Guesde s'appuient tous les deux sur un argumentaire socialiste, qui entérine le lieu commun de la lutte des classes, tout en identifiant des ennemis communs : le gouvernement bourgeois et la religion. La fonction de cette réunion n'est donc pas uniquement délibérative, mais aussi épidictique, dans la mesure où elle sert à réaffirmer un socle de valeurs préexistant. Ces valeurs font toutefois l'objet de stratégies de définition contradictoires, de manière à polariser les positions des deux intervenants. Cette scénographie de la contradiction implique, dans les deux discours, le développement d'une rhétorique polémique, dont les traits les plus récurrents sont (1) l'usage de pronoms inclusifs ou exclusifs à géométrie variable (on, nous, vous, eux), qui soutient souvent une dynamique d'inclusion/exclusion du locuteur et de son adversaire par rapport à l'auditoire, aux organisations partisanes ou militantes, au peuple (vs. la bourgeoisie/ le gouvernement), (2) la négation ou la dénonciation du discours de l'autre orateur, au 
moyen des différentes formes de discours rapporté. En ce qui concerne ce second trait, la structure de l'échange implique toutefois que seul l'orateur qui passe en second lieu puisse prendre appui sur le discours qui le précède ; celui qui passe en premier peut reprendre les dires défendus par son adversaire par le passé, dans une autre situation de communication. Dans les deux cas, les orateurs construisent leur ethos discursif en négociation avec leur propre ethos préalable, mais aussi avec celui de leur adversaire. La légitimation / délégitimation des idées passe en grande partie par la légitimation / délégitimation de la personne qui les défend.

\section{Guesde, Jaurès et la Gauche française en 1900}

Ce n'est pas le lieu ici d'exposer en détail les données biographiques et contextuelles qui entourent le corpus mis à l'étude. Nous nous limiterons à pointer, de manière volontairement synthétique, quelques éléments saillants utiles à la bonne compréhension des analyses qui suivront ${ }^{28}$.

13 La conférence de 1900 intervient juste après que les socialistes français se sont divisés sur deux importants dossiers, dont traitent les deux conférenciers. Le premier concerne la part prise par Jaurès dans les suites de l'Affaire Dreyfus, en 1898-1899 : les «Preuves» qu'il publie dans les journaux demandent la révision du procès, mais plaident aussi pour un vaste ralliement de tous les dreyfusards au courant socialiste. Le second concerne la "participation» de Millerand, socialiste indépendant, au cabinet ministériel de Waldeck-Rousseau, au sein d'un gouvernement «bourgeois » : Jaurès la défend, tandis que Guesde la condamne, au nom de la lutte des classes, ce qui provoque la scission de l'Union socialiste en septembre 1900, soit juste avant la conférence lilloise.

Sur ces dossiers, on peut dire que les deux figures que représentent Guesde et Jaurès s'opposent selon des lignes de partage idéologiques qui sont en même temps des éléments d'ethos importants pour saisir leur confrontation de 1900. Pour dire les choses caricaturalement, là où Guesde apparaît comme un pionnier du mouvement socialiste français, proche du radicalisme anarchiste, des grèves ouvrières et de l'objectif révolutionnaire, Jaurès appartient à la génération suivante (quatorze ans le séparent de Guesde). Son rapport au socialisme est davantage marqué par son activité intellectuelle (il soutient en 1892 une thèse sur les origines du socialisme allemand) et par sa foi en l'action politique parlementaire.

\section{L'analyse du discours socialiste : apports et limites}

L'émergence de l'analyse du discours $(\mathrm{AD})$ de tradition française est étroitement liée à une préoccupation pour le discours politique, pour sa spécificité par rapport aux autres types de discours, et pour la manière dont il cristallise des noyaux de sens idéologiques à fort impact collectif. Dès le numéro séminal de la revue Langages consacré à "L'analyse du discours» (1969), on trouve une importante contribution de cette discipline naissante à l'analyse du discours jaurésien ${ }^{29}$. Il s'agit de considérer le discours du leader socialiste comme l'une des matrices fondamentales et durables du discours politique en France, et de tenter de déterminer «les différentes opérations linguistiques qu'elle suppose de la part du sujet parlant ${ }^{30}$ ", en confrontant les énoncés «scientifiques" (les thèses philosophiques de Jaurès) et les énoncés «non 
scientifiques " (ses articles et discours engagés). La méthode d'analyse est directement inspirée de la linguistique distributionnelle de Z. Harris, qui consiste à «dresser des classes d'équivalence et à découvrir dans les segments successifs du texte des schèmes combinatoires de ces classes ${ }^{31} »$.

L'approche s'en tient donc prioritairement aux données (lexicales et syntaxiques) que fournit l'énoncé, et qu'elle traite quantitativement. Les hypothèses de travail sont construites à partir d'un champ sémantique déterminé a priori autour des noyaux lexicaux socialisme et socialiste, à l'exclusion des expansions "qui constituent des références socio-culturelles historiques, et celles qui renvoient par comparaison à des modèles socio-culturels littéraires ${ }^{32}$ ». On voit bien ainsi que l'interdiscours intervient comme un parasite possible du traitement quantitatif des données de l'énoncé.

Une dizaine d'années plus tard, la même revue consacre cette fois un dossier entier à "L'analyse linguistique du discours jaurésien ", qui republie l'article de 1969, assorti d'une plus copieuse étude, qui détaille l'évolution disciplinaire de l'AD et les problèmes théoriques et méthodologiques qu'elle a rencontrés, fournit une importante synthèse historique et biographique sur la trajectoire du locuteur Jaurès et entreprend l'analyse comparée de deux discours, tenus dans des contextes communicationnels différents. Les postulats méthodologiques n'ont guère évolué cependant; on peut même dire que le credo de scientificité affiché est encore accentué, notamment dans le souci d'isoler des invariants et des variables, auxquelles sont prêtées des fonctions explicatives ${ }^{33}$.

Cela dit, l'analyse entend intégrer davantage une nouvelle dimension: "les phénomènes d'énonciation, qui sont encore actuellement mal connus en linguistique. Ceux-ci affectent l'ensemble du discours, et non pas seulement des catégories particulières, telles que les embrayeurs ${ }^{34} »$. On voit bien que le caractère continu, et non discret, des phénomènes d'énonciation rend problématique leur étude dans un cadre méthodologique attaché à l'action de variables ; l'auteure confesse d'ailleurs ellemême: «Nous ne croyons pas [...] que l'analyse des phénomènes d'énonciation soit assez élaborée actuellement [...] pour rendre compte de la variation des formes de l'énonciation à un niveau aussi fin et aussi complexe que ceux qui viennent d'être mentionnés ${ }^{35}$ ». Sa conclusion finale réaffirme encore plus nettement : « ces problèmes d'énonciation, dont nous venons de suggérer les directions et de souligner brièvement l'importance, sont d'un bout à l'autre [d'une tout autre] complexité que ceux que soulèvent les simples énoncés informatifs dépouillés de leur sujet d'énonciation ${ }^{36}$.»

19 À la décennie suivante, Pierre Muller se penche quant à lui précisément sur le débat contradictoire entre Jaurès et Guesde, selon une perspective qu'on pourrait qualifier cette fois de radicalement énonciativiste. L'auteur se propose en effet d'étudier

[...] la manière dont chaque orateur désigne son adversaire et le public auquel il s'adresse, comment il s'insère lui-même, comment il situe les autres dans les différents groupes socio-politiques existants à cette époque [...]. Nous envisagerons donc successivement les mots que les deux orateurs utilisent pour se désigner l'un l'autre, puis le jeu des référents personnels ${ }^{37}$.

20 L'approche est une nouvelle fois quantitative et repose sur un traitement lexicométrique du corpus. La mise en évidence de deux grandes stratégies énonciatives (plutôt inclusive chez Jaurès, plutôt exclusive chez Guesde) est rapportée ultimement au " reflet d'une situation politique particulière ${ }^{38}$ ", à la position qu'y occupent les deux protagonistes et à la stratégie qu'ils y déploient, mais aussi à une «différence de tempérament » entre Jaurès et Guesde. 
21 Le même auteur revient, dans un dossier consacré à la négation en politique, sur le même corpus, envisagé cette fois par le biais du comptage des occurrences "susceptibles de nier une proposition ${ }^{39}$ ». Au-delà de cette approche quantitative, l'auteur reconnaît à son tour, après $\mathrm{G}$. Chauveau, que «[1]'étude systématique de tous les énoncés négatifs révèle en fait une grande complexité ", qui tient notamment au fait "que l'énonciateur n'est pas toujours le locuteur ${ }^{40}$ ». Par ailleurs, "[l]orsque l'énonciateur de la négation est l'auteur du discours, il faut essayer de déterminer à qui l'on peut attribuer l'énoncé affirmatif qui est ainsi rejetée ${ }^{41} »$.

Nous touchons là, nous semble-t-il, aux limites de l'approche quantitative, en même temps qu'aux enjeux mêmes d'une approche rhétorique soucieuse d'évaluer l'efficace d'un discours en prenant en considération à la fois sa situation de parole effective et la manière dont elle est réélaborée au sein des énoncés eux-mêmes. De même, l'approche énonciativiste ne peut, à nos yeux, se ramener au comptage des marques d'énonciation dans l'énoncé, en tant qu'elles refléteraient des paramètres extra-discursifs bien identifiables (relatifs au positionnement sociopolitique, voire au profil psychologique des locuteurs); elle doit plutôt rendre compte du feuilletage sémantique et argumentatif complexe qui se construit au gré des différentes voix qui émaillent un discours (des ethos préalables, des ethos discursifs, des énonciateurs délégués, des représentations de l'auditoire). Ces voix s'inscrivent elles-mêmes sur le fonds d'un imaginaire partagé et dans le cadre d'un rapport interlocutif particulier.

23 C'est, ainsi dit de manière très synthétique, le type de démarche que nous voudrions adopter dans l'analyse qui suivra, en considérant plus précisément que l'un des enjeux $\mathrm{du}$ débat contradictoire entre Guesde et Jaurès réside dans la manière dont les deux intervenants gèrent discursivement le rapport interlocutif, à la fois envers le contradicteur et envers l'auditoire (réel et imaginé).

\section{Analyse}

24 Notre analyse sera structurée de manière à mettre en exergue la méthode de Guesde par rapport à celle de Jaurès, essentiellement sur le plan de la gestion interdiscursive du rapport interlocutif. Pour le dire de manière schématique, alors que Jaurès, qui parle en premier, privilégie les jeux interdiscursifs pour eux-mêmes, ou pour leur fonction argumentative, en tout cas sans guère d'égards quant à leur intégration dans l'interaction concrète, Guesde utilise l'interdiscours pour encadrer son rapport à l'auditoire et à son contradicteur, pour y apporter des connotations, y convoquer des fragments d'imaginaire qui donnent un sens et une place aux partenaires de l'échange effectif.

25 Cette hypothèse générale se ventile selon deux grandes topiques, autorisant chacune des mouvements dialogiques internes par lesquels se construisent des positions énonciatives particulières : (1) la mémoire contre l'oubli, (2) le rapport de l'individu à la collectivité. 


\subsection{La mémoire contre l'oubli}

\subsection{1. « Je suis étonné, vraiment, d'avoir à rappeler ces vérités élémentaires »}

socialiste et la fraction la plus progressiste de la bourgeoisie, Jaurès s'étonne « d'avoir à rappeler ces vérités élémentaires »; il cite alors, littéralement, « Marx lui-même qui a écrit cette parole admirable de netteté: "Nous, socialistes révolutionnaires, nous sommes avec le prolétariat contre la bourgeoisie et avec la bourgeoisie contre les hobereaux et les prêtres" "(p.4). Un citoyen de l'assemblée interrompt aussitôt l'orateur pour dire : «Ce n'est pas vrai »; à quoi Jaurès répond : « vous vérifierez avec vos amis, nous vérifierons ensemble l'exactitude de la citation que j'ai faite et, si elle est exacte, je ne vous demanderai qu'une chose comme réparation : c'est, dans une de nos prochaines réunions, de venir en témoigner loyalement à cette tribune ${ }^{42}$. " (Ibid.) largement contre-productif dans le cadre de l'échange qu'il cherche à construire avec son public de militants. En voulant «rappeler » des " vérités élémentaires » pour tous les socialistes, et en convoquant pour cela la figure tutélaire de Marx sous la forme d'une citation littérale, il s'expose à ce que cette lettre même soit contestée ("Ce n'est pas vrai »), et à ce qu'il doive endosser l'habit du lettré. Le " vous vérifierez avec vos amis » instaure immanquablement une césure entre celui qui sait (citer de mémoire) et ceux qui doivent vérifier. Malgré la correction immédiate par «nous vérifierons ensemble", l'impression produite demeure que la réunion politique a adopté la scénographie de la salle de classe.

Un second épisode nous semble surenchérir sur cette scénographie et, plus douloureusement encore, sur les ratés qu'elle provoque dans l'interaction. Une nouvelle fois, il s'agit pour Jaurès de convoquer, sous la forme d'une citation littérale, un interdiscours chargé de resituer l'un des enjeux du débat de 1900 (la participation d'un socialiste à un gouvernement bourgeois) dans l'histoire longue du socialisme international. Plus précisément, Jaurès évoque l'exemplum du socialiste allemand Wilhelm Liebknecht, "l'admirable révolutionnaire » qui, en 1869, accepta d'entrer au Parlement et, dès lors, de prêter, devant Dieu, le serment de fidélité à la constitution royale et bourgeoise. Le passage entier, qui prend près d'une page, est un parfait exemple d'argument selon l'analogie historique, doublé d'un argument $a$ fortiori ; Jaurès cherche à montrer que les reproches qu'on adresse aujourd'hui à Millerand auraient pu être encore plus vifs en 1869, lorsque Liebknecht prêta son serment devant Dieu. Or, le 
déroulé de cet argument achoppe à l'endroit précis où Jaurès rapporte les paroles du socialiste allemand :

«Je jure devant Dieu » (Exclamations ironiques de plusieurs côtés).

Delory. - Citoyennes et citoyens, le besoin d'interruption place les interrupteurs dans une mauvaise posture puisque Jaurès n'a fait qu'une citation. (On rit).

Jaurès. - Mais, voyez, citoyens, à quel malentendu extraordinaire peuvent conduire les préventions que nous avons les uns contre les autres. Je viens de vous annoncer qu'on ne pouvait entrer au Landtag sans prêter un serment de fidélité au roi de Saxe; je vous rappelle, je vous décris la formule du serment prêté par Liebknecht pour entrer au Landtag de Saxe, et voilà des camarades un peu pressés qui ne sont pas fâchés de me taxer de cléricalisme. (Hilarité).

Représentez-vous bien que c'est Liebknecht qui parle : [...]. (p. 8)

L'extrait nous semble ici parfaitement représentatif des effets involontaires induits par le recours à un interdiscours à vocation mémorielle collective, qui en vient à activer, dans le rapport interlocutif, une scénographie que nous avons qualifiée de scolaire. Cette scénographie entre en conflit avec le rapport de places effectif entre l'orateur et son auditoire, de plusieurs manières. D'abord, le statut même de "discours rapporté " pour la citation du serment devant Dieu n'est pas parfaitement identifiable par l'ensemble de l'auditoire : c'est le fait d'entendre, in vivo, ces paroles dans la bouche du locuteur Jaurès qui prime sur le fait que ces paroles sont en réalité assumées par un énonciateur délégué. Ensuite, ce « malentendu » oblige Jaurès à expliciter sa manœuvre citationnelle, encore une fois selon un ethos d'instituteur face à une classe un peu récalcitrante. Enfin, l'épisode et la scénographie qu'il active partagent l'auditoire entre plusieurs sortes de rieurs : ceux qui rient de Jaurès citant le serment (les mauvais élèves, les " camarades un peu pressés »), et ceux qui rient des premiers rieurs (les bons élèves, qui ont compris d'emblée la manœuvre citationnelle et le mouvement argumentatif dans lequel elle s'inscrivait).

\subsection{2. « N'est-ce pas la vérité, Jaurès ?»}

Comme dit plus haut, l'ordre de passage des intervenants est déterminant dans la construction du discours. Guesde, qui passe en deuxième, peut prendre appui sur le discours de son adversaire qui le précède, le citer et le juger, alors que Jaurès, lui, n'aura pas de droit de réponse. Bien conscient de cet avantage, Guesde se place d'emblée en juge de la véridicité des propos qui viennent d'être tenus par Jaurès. Les trois premiers jugements sont positifs («remercier Jaurès d'avoir aussi bien posé la question », «Jaurès a dit la vérité [...] lorsque », « Jaurès a eu raison également, lorsqu'il [...] ») ; ils valident l'accord de Guesde sur trois des prémisses évoquées par Jaurès : les deux points de dissensus (Dreyfus et Millerand) et la définition de la lutte des classes, socle de valeurs communes qui fonde la dimension épidictique du débat. Cette entrée en matière est très brève et le reste du discours est construit sur le mode du désaccord. De multiples citations des paroles de Jaurès sont ainsi accompagnées de commentaires les jugeant négativement («il a été très imprudent en invoquant»), en dénonçant les oublis («Ce qu'il ne vous a pas dit», «Jaurès oubliait de vous dire que...»), ou les mettant en rapport avec des lieux communs répulsifs, comme par exemple la religion ( « de telle façon qu'il assimilait la lutte de classe au paradis des chrétiens et des catholiques »).

Ce faisant, Guesde se pose comme celui qui vient rétablir la vérité dans le discours de Jaurès, en complétant les éléments que ce dernier aurait passés sous silence ( « on a fait 
appel à des souvenirs personnels, je demande à les compléter ", p. 12). Trois souvenirs sont en particulier corrigés: (1) le débat à la Chambre des députés à la fin de la législature de 1893-1898, (2) le scandale provoqué par un chef d'état-major et des galonnés supérieurs à propos de Zola, (3) le conseil des socialistes organisé par Millerand et Viviani, avec Vaillant, Guesde et Jaurès avant la publication de la Déclaration du Conseil national du POF (le Parti Ouvrier Français). Guesde prend particulièrement soin de rappeler quelles ont été les paroles tenues par lui-même et par les différents acteurs de ces discussions au moyen de discours directs, mobilisant ainsi une rhétorique du témoignage qui se présente comme reproduisant exactement les faits.

34 Alors qu'il se place lui-même dans le rôle du juge de la véracité des propos de Jaurès, Guesde demande à ce dernier de confirmer la véracité de ses propos à lui : « Est-ce vrai, Jaurès ? N'est-ce pas le langage que j'ai tenu ? ", « Est-ce vrai encore, citoyen Jaurès ? " (p.11), "Jaurès reconnaît que ce que je rapporte est l'exacte vérité»(p.12). Il se montre ainsi comme ne craignant pas la contradiction et comme effectuant une démarche que Jaurès n'a pas faite, mais aurait dû faire. Loin de donner une légitimité à son adversaire, Guesde entend par ces interlocutions réclamer de celui-ci une soumission. Car en effet, en répondant favorablement à la requête de Guesde ("C'est très bien, c'est très juste », p. 11, «Je l'ai toujours dit », p. 12), Jaurès n'affirme pas seulement que Guesde dit vrai, indirectement il se rétracte et admet l'incomplétude de son propre propos.

En outre, les discours directs cités par Guesde pour corriger les souvenirs évoqués par Jaurès sont généralement des actes de parole par lesquels il se met lui-même au premier plan des évènements racontés, tandis que Jaurès est cantonné au rôle de spectateur, ou de la personne à qui les autres s'adressent: "c'est alors que j'ai protesté » (p. 10), «j'ai protesté », «j'ai dit autre chose encore », «j'ai été plus loin : j’ai dit que [...], j'ai dit qu'il [...]», «j'ai dit à Jaurès que [...], j'ai ajouté [...]» (p. 11), etc. À propos de l'affaire Dreyfus en particulier, Guesde se forge l'image du militant qui proteste seul contre tous les modérés (p. 10-11). Dans ce cadre, les questions du type «Est-ce vrai, Jaurès? » qui ponctuent le discours de Guesde ont surtout pour fonction de forger de l'interlocuteur l'image d'un témoin passif des échanges, seulement apte à attester de la véracité ou non des paroles qui ont été tenues par Guesde, y compris dans les débats concernant l'affaire Dreyfus.

Cette adresse de Guesde à Jaurès est également dirigée vers un interlocuteur tiers, le public. Il s'agit de susciter chez ce dernier une suspicion quant à la véracité des propos qui, de manière générale, peuvent être tenus lors d'une réunion politique. Guesde convoque ainsi deux paires d'imaginaires - la vérité contre le mensonge et la mémoire contre l'oubli - qu'il organise de manière à composer une scénographie où son propre ethos discursif s'oppose axiologiquement à l'image qu'il donne de son adversaire.

Outre le fait que Guesde oblige Jaurès à revenir sur ses propres propos - donnant de ce dernier une image versatile, peu digne de confiance -, Guesde convoque le lexique du mensonge et de la duperie lorsqu'il décrit la manière dont Jaurès interprète la notion de lutte des classes :

La lutte de classe, telle que l'a très bien définie Jaurès, si elle ne devait pas déterminer votre conduite de tous les jours, la politique de la classe ouvrière, la tactique nécessaire du prolétariat organisé en parti de classe, serait un mensonge et une duperie [...]. (p. 10) 
loin, Jaurès est présenté comme un négociateur tentant de faire conclure au prolétariat « un marché de dupes » (p. 15). Par opposition, Guesde construit son ethos sur l'image de la stabilité et de l'opinion inflexible :

Nous ne reconnaissons pas la lutte de classe, nous, pour l'abandonner une fois reconnue, une fois proclamée ; c'est le terrain exclusif sur lequel nous nous plaçons, sur lequel le Parti ouvrier s'est organisé, et sur lequel il nous faut nous maintenir pour envisager tous les évènements et pour les classer. (p. 10)

Lorsque notre Déclaration a paru, elle ne faisait donc que maintenir une décision qui avait toujours été la nôtre et qui exprimait la volonté concordante des différentes fractions socialistes. (p. 12)

En couplant la dichotomie mensonge/vérité à celle de la versatilité contre la stabilité Guesde mobilise stratégiquement les images doxiques de son ethos préalable et de celui de son adversaire : fondateur du POF, Guesde incarne la genèse du parti ouvrier. Son passé est derrière lui et la reconnaissance de l'auditoire lui est acquise. Bref, « il a fait ses preuves ». Comme dit plus haut, Jaurès est quant à lui un nouveau venu au socialisme ouvrier. Sur cette base, Guesde peut assez facilement inspirer la méfiance du public envers Jaurès qui entend «d'ores et déjà » infléchir la ligne directrice du mouvement ouvrier dans une négociation avec le gouvernement bourgeois.

$\mathrm{Au}$ mensonge et à la versatilité, Guesde ajoute encore l'oubli. Il souligne l'omission de certains détails dans les souvenirs rapportés par Jaurès ( Jaurès oubliait de vous dire que ... ", «J'en appelle encore une fois à la mémoire de Jaurès ») et utilise le lexique de l'oubli pour décrire l'attitude que ce dernier prescrit au prolétariat :

On nous a dit : La lutte de classe existe ; mais elle ne défendait pas, elle commandait au contraire au prolétariat, le jour où une injustice avait été commise, le jour où une condamnation inique était venue atteindre un membre de la classe dirigeante, elle faisait un devoir, une loi aux travailleurs, d'oublier les monstruosités qui se perpètrent tous les jours contre leurs familles, contre leurs femmes et contre leurs enfants.

Ils devaient oublier tout cela ; [...] (p. 10)

41 Ce faisant, Guesde dresse l'image d'un prolétariat dénaturé parce que contaminé par l'oubli de Jaurès. Il construit de nouveau son propre ethos discursif en contre-pied, se présentant comme le gardien de la mémoire du prolétariat, celui qui complète les souvenirs: «[...] je croyais qu'il fallait être prêt à piétiner le prétendu ordre d'aujourd'hui pour faire ainsi place à l'autre », " je croyais, moi, qu'ils avaient marché contre ce régime, qu'ils avaient tout balayé, le mauvais et le bon, le bon avec le mauvais ; et je croyais que le prolétariat ne serait pas moins révolutionnaire » (p. 13).

Tout en se faisant le porte-étendard individué des croyances révolutionnaires passées, Guesde construit face à lui une image de la «nouvelle politique » défendue par Jaurès, en l'attribuant à des tournures ou pronoms impersonnels : « Il paraît que non; il paraît que les grands bourgeois de 1789 auraient dû se préoccuper de défendre l'ancien régime, sous prétexte des réformes réalisées au cours du dix-huitième siècle ", " La nouvelle politique que l'on préconise au nom de la lutte de classe consisterait donc à organiser à part, sur son propre terrain, le prolétariat [...]» (p.13). À la perte des anciens idéaux s'ajoute ainsi une désincarnation de cette nouvelle politique : l'usage du « il » impersonnel tend à déposséder Jaurès de la politique qu'il défend, la faisant appartenir à un collectif indéfini, de manière à attiser la méfiance du peuple. Encore une fois, cette dichotomie mémoire/oubli, couplée à celle du «je » contre le «il» indéfini, s'appuie sur les ethos préalables des deux orateurs : Guesde est la mémoire du 
parti et du prolétariat, il est une figure emblématique, connue, face à Jaurès arrivé tardivement: la politique qu'il défend est présentée comme ne lui appartenant pas directement. On peut voir là une dénonciation implicite des liens de Jaurès avec Millerand.

Les valeurs d'authenticité, de mémoire et de maintien d'une même ligne idéologique sont ainsi présentées comme étant celles à l'aune desquelles l'auditoire doit guider son jugement et son choix d'une stratégie politique à suivre. Guesde se présente comme l'incarnation de ces valeurs face à Jaurès présenté comme leur antithèse. La dimension délibérative du discours de Jaurès est ainsi détournée et invalidée par la dimension épidictique du discours de Guesde.

Le public se voit conférer un rôle dans cette stratégie discursive. Guesde ne qualifie pas seulement les paroles de Jaurès, mais l'interlocution de ce dernier avec le public; l'interlocution citante requalifie ainsi l'interlocution citée, le public, identique, désigné à la deuxième personne du pluriel, sert de pivot entre ces deux échanges : «Jaurès a eu raison également, lorsqu'il a commencé par vous fournir [...]», «lorsqu'il vous a rappelé ", "c'était en vous plaçant ", "il vous l'a très bien définie ", "il vous l'a montrée ", "il vous a dit » (p. 10). Par cette surénonciation ${ }^{43}$ s'adressant au même public que l'énonciation citée, Guesde se pose comme le gardien du prolétariat et plus spécifiquement comme le garde-fou permettant de filtrer les mensonges et de corriger les erreurs et oublis véhiculés par Jaurès au public.

\subsection{L'individu et la collectivité}

\subsection{1. « II faut que vous sachiez »}

45 L'une des principales lignes argumentatives de Jaurès pour défendre la participation socialiste au gouvernement bourgeois consiste à opérer un distinguo entre "le principe » de la lutte des classes et «la tactique » qui doit orienter l'action politique concrète au jour le jour. Nous avons déjà évoqué plus haut le second de ces volets, qui repose pour l'essentiel sur le recours aux exempla. Quant au premier, il place Jaurès dans une énonciation de type théorisant et didactique qui va en réalité constituer la marque dominante de tout son discours ${ }^{44}$. Cette dominante situe la topique du rapport de l'individu à la collectivité sur le terrain du savoir et du devoir.

Jaurès ponctue en effet son exposé sur le principe de la lutte des classes d'une série de "il faut», qui introduisent les conditions requises pour une émancipation du prolétariat. Si elles sont présentées comme toutes théoriques, ces conditions n'en produisent pas moins un effet d'injonction, qui est inséparablement une injonction à connaître lesdites conditions et une injonction à les incarner effectivement. Ce lien construit entre un savoir théorique et un devoir pratique est renforcé par les nombreuses occurrences du mot «devoir», associé systématiquement au "prolétariat» ou aux « socialistes ${ }^{45} »$ : « il était du devoir des socialistes », «c'est le devoir des socialistes », «c'est le devoir du prolétariat socialiste », « il est impossible au prolétariat socialiste, sans manquer à tous ses devoirs", "c'était le devoir du prolétariat », "le prolétariat a doublement rempli son devoir envers lui-même ». La conception qui est donnée du prolétariat et de la lutte des classes est formulée sur le mode de la définition d'essence et s'accompagne d'implications déontiques. 
Dans une telle configuration discursive, le rapport $\mathrm{du}$ « je » au « nous » est placé sous la coupe d'un «il faut» impersonnel. On trouve certes de nombreux «je vous dis", " laissez-moi vous dire ", " nous savons ", " vous savez ", dans le discours de Jaurès, qui parle aussi de «ma démonstration" pour qualifier son propos, mais ces occurrences insistent en réalité moins sur les personnes engagées que sur les verbes eux-mêmes et sur les modalités du débat politique auxquelles ils renvoient. La première injonction sur laquelle insiste Jaurès dès les premiers mots de sa conférence, et qui va colorer l'ensemble de son propos, c'est celle invitant les militants au calme: «Le plus grand plaisir que vous puissiez nous faire, ce n'est pas de nous acclamer, c'est de nous écouter.» (p.1) Plus loin, il invite à aborder telle question «avec calme» (p.6), en privilégiant désormais les arguments plutôt que les « cris variés » (ibid.).

Autrement dit, l'échange avec l'auditoire est en quelque sorte jaugé à l'aune de l'idéal auquel il devrait correspondre, et ainsi constamment réajusté en fonction de ce que les partenaires de l'échange devraient savoir. Ainsi, même lorsqu'il est question de la «tactique» et du positionnement individuel de Jaurès dans l'affaire Millerand, l'orateur introduit son évocation par la locution «Il faut que vous sachiez », qui, en résonance avec les « il faut » de l'exposé théorique, nous semble ici pointer l'horizon de savoir commun auquel devraient tendre les militants.

49 À cette modalité majoritairement déontique, par laquelle se construit chez Jaurès le rapport de l'individu à la collectivité, Guesde va substituer une (double) lecture situant quant à elle cette même topique sur le fonds d'un imaginaire de l'héroïsme d'une part, et de la trahison d'autre part.

\subsection{2. " Je dis que nous » : I'individu pour la collectivité}

50 Lorsqu'il s'agit d'affirmer le maintien de la ligne idéologique du mouvement ouvrier, Guesde prend la parole au nom de collectifs - le POF, les signataires de la Déclaration -, en employant le pronom inclusif «nous». Néanmoins, il s'exprime encore plus fréquemment en «je», de sorte que le «je» devient souvent le surénonciateur du «nous ». Dès la première section du discours, on observe l'usage de boucles réflexives pour désigner les actes de parole des deux orateurs (lui-même et Jaurès) : «Seulement, à mon avis, il a été très imprudent en invoquant ce qu'il appelle un principe, et ce que j'appelle, moi, un fait : la lutte de classe (p. 10)».

51 La lutte des classes est une notion entérinée et validée par la doxa socialiste de l'époque; socle de valeurs partagées, elle sert de prémisse au débat et l'auditoire n'a pas à la remettre en question. Un choix est appelé à être posé entre deux interprétations différentes de l'usage à faire de cette notion. Dans ce cadre, la mention des actes de parole, individués par leur attribution à des pronoms singuliers - «il appelle » vs. «j'appelle »-, sous la forme de deux boucles réflexives ${ }^{46}$, remplit au moins deux fonctions liées entre elles.

D'une part, l'orateur se confère à lui-même ainsi qu'à son adversaire la légitimité de redéfinir le rôle que doit prendre cette notion doxique dans l'action concrète et la prise de décision de l'auditoire-peuple ; ce faisant, il légitime sa capacité à prendre la parole publiquement, à interpréter les références communes, à les renommer et à formuler les décisions politiques à prendre, par opposition à l'auditoire-peuple auquel n'est reconnu aucun pouvoir d'interprétation, ni laissé aucune possibilité de prise de parole, concernant une notion ( « principe » ou « fait ») qui fait pourtant partie des idéaux pour 
lesquels il a décidé de se battre. Ce dispositif active l'image, doxique elle aussi, d'un peuple passif et silencieux. Cette image du peuple est aussi activée plus loin, lorsque Guesde parle du prolétariat organisé et du prolétariat non organisé en les désignant respectivement comme « les cerveaux conquis » et « les cerveaux d'à côté où la lumière socialiste ne s'est pas encore faite » (p. 14).

D'autre part, en utilisant des pronoms singuliers (Guesde aurait pu dire "nous»), l'orateur fait entrevoir la nécessité du régime représentatif, tout comme la nécessité de la confrontation politique, qui toutes deux participent de sa raison d'être. Ces boucles réflexives sont donc mises au service d'une autolégitimation de son rôle d'orateurreprésentant, mais aussi de celui de son adversaire.

Dans cet ordre d'idées, l'entièreté du discours de Guesde est jonchée de verbes de parole à la première personne. Comme dit plus haut, dans son récit de l'affaire Dreyfus en particulier, Guesde s'autocite en se représentant dans une scénographie du « seul contre tous »: "C'est contre cette lâcheté électorale, contre ces hommes qui ne pensaient qu'à leur siège de député que j'ai protesté »(p.11). Ici c'est bien en un individu - lui-même - que Guesde intime à l'auditoire de croire ; il se forge de lui-même l'image du seul véritable porte-parole politique des intérêts du peuple face aux modérés qualifiés d'opportunistes. Il s'institue également en guide de son propre mouvement, comme le montrent les extraits suivants :

(1) [...] l'intérêt de la classe ouvrière que je représentais (p. 11)

(2) Lorsque notre Déclaration a paru, elle ne faisait donc que maintenir une décision qui avait toujours été la nôtre [...]. Je dis que nous ne pouvons reconnaître à la bourgeoisie, lorsqu'une injustice frappe un des siens, le droit de s'adresser au prolétariat [...] (p. 11-12)

(3) [...] il aurait fallu [...] coudre le prolétariat à cette queue de la bourgeoisie emprisonneuse [...]

(4) Ah non! camarades. À ce moment-là, le Parti ouvrier a crié : Halte-là ! (p. 12)

(5) Je me souviens d'un parti républicain dont j'ai été, le vieux parti républicain, qui se refusait au genre de compromission que l'on voudrait imposer aujourd'hui à notre Parti socialiste (p. 14).

Il ressort de tous ces extraits un jeu subtil d'oscillation du locuteur entre le « je » et le «nous », entre le représentant individuel et le représentant collectif du prolétariat. En (1), Guesde envisage la représentation du prolétariat dans une logique individuelle plutôt que dans une logique de parti ou de mouvement. En (2), à la décision prise collectivement depuis "toujours", se surajoute l'impératif posé par Guesde à la collectivité, dans le présent de l'interlocution: «je dis que nous...». En (3), l'interjection et l'expression du refus de Guesde au présent de l'interlocution («Ah non! camarades ») précède l'interjection poussée par le Parti ouvrier au moment des faits rapportés (« Halte-là ! »). En (4) enfin, Guesde s'institue comme le détenteur de la mémoire face à la collectivité menacée par l'oubli (voir supra). Tous ces mécanismes vont dans le sens d'une autolégitimation par Guesde de son rôle de représentant individuel du prolétariat, au-dessus de la logique de parti, en se donnant une fonction de pivot entre le passé et le présent, exploitant en quelque sorte l'image doxique du vieux sage.

\subsection{3. « vous ne pouvez pas engager le Parti » : l'individu contre la collectivité}

Une transition subtile va permettre à Guesde de faire ensuite paraître le positionnement réfractaire de Jaurès par rapport à une collectivité politique, non pas 
comme un positionnement fort et héroïque (comme pour lui-même), mais comme une forme de trahison. Cette transition s'opère par la convocation de l'image de Dreyfus, victime unique derrière laquelle Jaurès ambitionne d'engager tout le prolétariat et le socialisme. Guesde rend cette position intenable en donnant de Dreyfus l'image d'un homme auquel le prolétariat ne peut pas s'identifier :

Ah! je comprends bien que vous, les ouvriers, vous, les paysans, que l'on arrache à l'atelier, que l'on arrache à la charrue, pour leur mettre un uniforme sur le dos [...], vous ayez le droit et le devoir de crier vers nous, vers le prolétariat organisé, lorsque vous êtes frappés par cette épouvantable justice militaire, parce que vous n'êtes pas à la caserne de par votre volonté, parce que vous n'avez jamais accepté ni les règlements, ni l'organisation, ni la prétendue justice militaire que vous subissez ; mais lui, il savait ce qu'il avait devant lui lorsqu'il a choisi le métier des armes; c'est de propos délibéré qu'il s'est engagé dans cette voie, partisan des conseils de guerre tant qu'il a cru qu'ils ne frapperaient que les prolétaires et que c'est lui, dirigeant, officier, qui mettrait contre eux en mouvement cette justice aveugle et à huis clos. Telle était la victime pour laquelle on avait la prétention de mobiliser tout l'effort prolétarien et socialiste... (p. 11-12).

De nouveau, les pronoms «vous » et «nous » sont utilisés pour (1) mettre à distance ceux qui seraient tentés de s'identifier à Dreyfus en raison de l'injustice militaire qu'ils subissent également, et (2) inclure les prolétaires organisés et sa propre personne dans un même groupe vers qui se tourne le prolétariat trahi. Dreyfus est présenté comme la victime du système qu'il contribuait à faire fonctionner contre les prolétaires. Cette image de bourreau des prolétaires a pour fonction de rendre inconcevable au prolétariat la volonté de venir en aide à Dreyfus.

L'image du capitaine comme victime isolée et indigne du prolétariat ( il y a une victime particulière qui a droit à une campagne spéciale et à une délivrance isolée ; cette victime-là, c'est un des membres de la classe dirigeante, c'est un capitaine d'étatmajor ») ouvre une voie propice à la construction d'une image négative de Jaurès, celle d'un individu perturbateur, qui à lui seul, pour un seul (Dreyfus), désobéit au concile socialiste en engageant toute la collectivité :

[...] il ne s'agissait pas surtout d'imposer au prolétariat le salut d'un homme à opérer, lorsque le prolétariat a sa classe à sauver, a l'humanité entière à sauver ! (p. 11)

[...] au bout de l'affaire Dreyfus, il n'y a pas eu de suppression des conseils de guerre, il n'y a pas eu la moindre modification à la justice militaire, il n'y a rien eu de ce qu'on vous promettait ; il y a eu un homme qui a été arraché à son rocher de lîle du Diable; campagne personnelle, elle n'a eu qu'un résultat personnel. (p. 12-13)

Oh! je me trompe, il y a eu quelque chose, et ce quelque chose, c'est Jaurès luimême qui a eu le courage de le confesser. Il vous a dit : De l'affaire Dreyfus, [...] il est sorti la collaboration d'un socialiste à un gouvernement bourgeois. [...] cette nouvelle forme de coopérative réunissant dans le même gouvernement un homme qui, s'il est socialiste, doit poursuivre le renversement de la société capitaliste, et d'autres hommes, en majorité, dont le seul but est la conservation de la même société, on nous la donne comme un triomphe du prolétariat, comme indiquant la force acquise par le socialisme. (p. 13)

On observe ici une variante du lieu « seul contre tous », qui serait plutôt ici « l'individu contre la collectivité », décliné en trois avatars, Jaurès, Dreyfus et Millerand, jugés cette fois négativement, le destin de tous ne pouvant être attaché à la cause ou au travail d'une seule personne. 

en symétrie avec celle de Dreyfus décrite plus haut. Guesde rappelle en effet que parmi les dreyfusards figuraient des dirigeants qui, comme Trarieux et Guyot, n'ont "pas craint de frapper sans jugement, tous les ouvriers et employés des chemins de fer [...]» (p. 12).

61

Pour construire son ethos en opposition à l'individualisme ici dénoncé, Guesde affirme à propos de lui-même, concernant le fait qu'il ne se soit pas défendu contre les accusations qui lui étaient faites d'être «vendu à Dreyfus » :

Non, camarades, pas plus alors que jamais, je ne me suis préoccupé des conséquences personnelles que pouvaient avoir mes actes, qui ont toujours été dirigés, déterminés, commandés par l'intérêt de la classe ouvrière que je représentais, - et que j'entendais représenter seule [...]. (p. 11)

De cette manière, Guesde dénonce implicitement le positionnement de Jaurès comme étant motivé par des intérêts personnels. Cette dénonciation implicite se manifeste également dans le passage « $\mathrm{Oh}$ ! je me trompe, il y a eu quelque chose, et ce quelque chose, c'est Jaurès lui-même [...]». Même si la phrase se poursuit avec le «quelque chose ", renvoyant à Millerand, la linéarité du discours oral tend à imprimer, dans l'esprit de l'auditoire destinataire, un rapport fort entre ce "quelque chose» et le syntagme qui suit immédiatement: "Jaurès lui-même ». Ainsi, tout en validant la logique représentationnelle et le rôle des orateurs comme porte-paroles et guides du peuple, Guesde met cependant son auditoire en garde contre les politiciens qui voudraient engager la collectivité dans des combats qui n'auraient en réalité qu'une finalité personnelle.

\subsection{Bilan de l'analyse}

63 Ce qu'il ressort de ces différents mouvements dialogiques, c'est, qu'en somme, l'image du peuple construite par Guesde dans ce discours est moins liée à des thèses ou à des contenus idéologiques qu'à un certain rapport à la parole dans l'espace public. Autant les actes de parole des représentants politiques sont surreprésentés, légitimant ainsi le régime représentatif, autant le public est rendu passif : il est amené à se décider entre deux méthodes, mais ce qui, selon Guesde, doit avant tout guider son choix, c'est le devoir d'authenticité, de mémoire, de conformité à la ligne idéologique suivie depuis le début par le parti. Comme dit plus haut, la délibération est donc soumise à l'épidictique. Cette passivité imposée est également visible dans la manière dont Guesde réagit aux rares interruptions du public :

Ah! camarades, on a fait appel à des souvenirs... (Applaudissements.) oh! n'applaudissons pas, je vous prie, laissez-moi aller jusqu'au bout sans ajouter, par vos bravos, à ma fatigue. (p. 12)

64 Guesde se dresse ainsi en figure d'autorité, non seulement face à son adversaire dont il démonte le discours tout en exigeant de lui qu'il avoue et confirme ses erreurs, mais aussi face au public qu'il enjoint au silence - prenant ainsi momentanément le rôle du modérateur Delory - et auquel il dicte les valeurs et la conduite à suivre.

Les réactions retranscrites tendent à montrer que le public se conforme au rôle qui lui est assigné par Guesde. Les interruptions sont très rares et sont principalement constituées par des «Bravos!» et des applaudissements. Un «Non! Non!» (p. 11) atteste de la vérité du témoignage de Guesde et une mention de «Rires et 
applaudissement» (p.12) va également dans le sens d'une adhésion au point de vue de Guesde, les rires arrivant en réponse à la représentation grotesque que Guesde donne des bourgeois dreyfusards - rendue sans doute encore plus grotesque par l'emploi du subjonctif imparfait dans le discours indirect : «il aurait fallu que même les serfs des voies ferrées oubliassent le crime projeté contre leur classe pour faire cause commune avec M. Trarieux, avec M. Yves Guyot, avec la fine fleur des bourgeois exploiteurs » (p. 12). Ce subjonctif imparfait, comme insigne verbal du langage recherché de la classe dirigeante, en contraste avec les expressions «serfs » et «fine fleur des bourgeois exploiteurs ", qui renvoient quant à elles à de tout autres points de vue énonciatifs, témoigne chez Guesde d'une grande maîtrise des voix convoquées dans le discours, des imaginaires associés à ces voix, et de la position du peuple par rapport à ces imaginaires. C'est sans doute là l'une des différences fondamentales qu'il faut pointer avec le discours de Jaurès, dans lequel le rapport interlocutif avec l'auditoire vient perturber la lisibilité des jeux interdiscursifs.

\section{NOTES}

1. P. Muller, «Jaurès et Guesde: deux interlocuteurs socialistes en 1900 », Mots, 19, 1989, p. 53-65 ; P. Muller, «Jaurès et Guesde : quand une méthode en nie une autre », Mots, 45/1, 1995, p. 10-22.

2. P. Cossart, Le meeting politique. De la délibération à la manifestation (1868-1939), Rennes, Presses universitaires de Rennes, 2010.

3. Ibid., p. 10-15, 84.

4. R. Chartier, Les origines culturelles de la Révolution française, Paris, Seuil, 1990, p. 41, cité dans Cossart, op. cit., p. 16.

5. P. Cossart, op. cit., p. 16.

6. Ibid., p. 74.

7. Ibid., p. 19.

8. Voir W. Reddy, The Navigation of Feeling, A Framework for the History of Emotions, New York, Cambridge UP, 2001, p. 323, cité dans P. Cossart, op. cit., p. 19.

9. Y. Déloye \& O. Ihl, «La civilité électorale : vote et forclusion de la violence en France », Cultures \& conflits, 9-10/75-76, 1993, p. 75-76, cité dans P. Cossart, op. cit., p. 19.

10. Voir N. Elias, La Civilisation des mœurs, Paris, Calmann-Lévy, 1973, cité dans P. Cossart, op. cit., p. 20.

11. P. Cossart, op. cit., p. 48.

12. Ibid., p. 20.

13. Ibid., p. 76.

14. Ibid., p. 84-88.

15. Ibid., p. 89.

16. Ibid., p. 90-91.

17. Ibid., p. 92.

18. Ibid., p. 92-93. 
19. Voir N. Rousselier, "La diffusion de l'éloquence en France sous la III ${ }^{\mathrm{e}}$ République ", dans F. D’Almeida (dir.), L'Éloquence politique en France et en Italie de 1870 à nos jours, Rome, École française de Rome, 2001, p. 41-46, 45.

20. Cité d'après P. Cossart, op. cit., p. 97.

21. Ibid.

22. Voir M.-L. Degrenne, Les réunions et les pouvoirs de police, thèse de droit, Caen, Caron \& Cie, 1938, p. 205, cité dans P. Cossart, op. cit., p. 97.

23. Voir A. Joubrel, Du droit de réunion, Rennes, Librairie générale Pilhon et Hommay, 1904, p. 149, cité dans P. Cossart, op. cit., p. 97.

24. C. Constant, Code des réunions publiques, des réunions électorales et des réunions privées, Commentaire pratique de la loi du 30 juin 1881 à l'usage des préfets, sous-préfets, maires, juges de paix, ainsi que des organisateurs de réunions publiques ou privées, Paris, A. Durand et Pedone-Lauriel, 1881, p. 60, cité dans P. Cossart, op. cit., p. 97-98.

25. Louis Jourdan, Le Siècle, 14 mai 1868, cité dans P. Cossart, op. cit., p. 99.

26. P. Cossart, op. cit., p. 100.

27. Ibid., p. 102.

28. L'analyse du discours de Jaurès par G. Chauveau propose une longue section historique qui fournit une synthèse très éclairante de la trajectoire biographique et politique de Jaurès. Voir G. Chauveau, « Analyse linguistique du discours jaurésien », Langages, 52, 1978, p. 7-109.

29. G. Provost, "Approche du discours politique: "socialisme" et "socialiste" chez Jaurès", Langages, 4/13, 1969, p. 51-68.

30. Ibid., p. 52.

31. Ibid., p. 53.

32. Ibid., p. 52.

33. Voir notamment la «Présentation » au dossier, donnée par Jean Dubois.

34. G. Chauveau, « Analyse linguistique du discours jaurésien », op. cit., p. 35.

35. Ibid., p. 36-37.

36. Ibid., p. 106.

37. P. Muller, « Jaurès et Guesde : deux interlocuteurs socialistes en 1900 », op. cit., p. 56.

38. Ibid., p. 63.

39. P. Muller, " Jaurès et Guesde : quand une méthode en nie une autre », op. cit., p. 11.

40. Ibid., p. 15.

41. Ibid., p. 16.

42. Selon ce qu'indiquent les éditeurs des œuvres de Jaurès, "Jaurès cite de mémoire, de façon approximative, une phrase de l'avant dernière page du Manifeste: "En Allemagne, le parti communiste luttera aux côtés de la bourgeoisie dans toutes les occasions où la bourgeoisie reprendra son rôle révolutionnaire ; avec elle, il combattra la monarchie absolue, la propriété foncière féodale, la petite bourgeoisie" (K. Marx et F. Engels, Le Manifeste communiste, trad. de Ch. Andler, [Société nouvelle de librairie et d'édition, Librairie Georges Bellais, 1901], p. 73.)» (Jean Jaurès, Euvres, tome VIII, «Défense républicaine et participation ministérielle (1899-1902)», édition établie par Maurice Agulhon et Jean-François Chanet, Paris, Fayard, 2013, note 3, p. 323.)

43. Voir A. Rabatel, «Ironie et sur-énonciation », Vox Romanica, 71, 2012, p. 42-76.

44. Sur « l'intégration du schème didactique à l'intérieur de l'énoncé politique », voir G. Provost, op. cit., p. 63-65.

45. Sur la fréquence des modalisations avec devoir dans l'ensemble du discours politique de Jaurès, voir G. Provost, op. cit., p. 59-60.

46. Voir J. Authier-Revuz, Ces mots qui ne vont pas de soi. Boucles réflexives et non-coïncidences du dire, rééd., Limoges, Lambert-Lucas, « Linguistique », [1995] 2012. 


\section{AUTEURS}

\section{ÉMILIE GOIN}

Université du Luxembourg

\section{FRANÇOIS PROVENZANO}

Université de Liège 